\title{
About Boltzmann Equations for Transport Modeling in Semiconductors
}

\author{
F. Poupaud \\ Laboratoire J.A. Dieudonné, URA 168 du CNRS Université de Nice \\ Parc Valrose, F-06108 Nice Cédex, FRANCE
}

\begin{abstract}
We present some results on the mathematical analysis of kinetic equations for modelling transport processes in semiconductors. We focus our attention on the connection between the kinetic models and the fluids ones based on driftdiffusion or hydrodynamic equations. Asymptotic analysis gives hydrodynamic coefficients in terms of microscopic quantities and allows to derive accurate boundary conditions.
\end{abstract}

\section{Introduction}

Transport modelling in modern submicronic devices requires a kinetic or quantum description. It explains the increase of interest in numerical simulation and mathematical analysis of the Boltzmann equations for semiconductors.

Perhaps one of the major issue for modelling is to couple simulations on the three levels : quantum, kinetic and fluids, depending on the regions of the device. Doing this requires a very precise description of the mathematical connection between this three levels. We present here some result on this topic.

2. Existence theory for kinetic models and connection with quantum physics.

One of the most general kinetic models is based on the following Boltzmann equation, [1] :

$$
\partial_{t} f+v(k) \cdot \nabla_{x} f-\frac{q}{\hbar} E \cdot \nabla_{k} f=Q(f),
$$

where the distribution function $f$ depends on time $t$, position $x$ and wave vector $k$. The electric field is denoted by $E$ and satisfies the Poisson equation. Therefore it depends on the distribution $f$ through the concentration $n$ :

$$
n(t, x)=\int f(t, x, k) \frac{d k}{4 \pi^{3}} .
$$


Therefore the Boltzmann equation is nonlinear. Moreover if no degeneracy assumption is made, the collision operator reads :

$$
Q(f)=\int s\left(k^{\prime}, k\right) f(k)\left(1-f\left(k^{\prime}\right)\right)-s\left(k, k^{\prime}\right) f\left(k^{\prime}\right)(1-f(k)) d k^{\prime} .
$$

Thus it is also nonlinear. Above, $s$ is the scattering rate. From the principle of detailed balance, it follows that:

$$
s\left(k^{\prime}, k\right) M(k)=s\left(k, k^{\prime}\right) M\left(k^{\prime}\right)
$$

where $\mathrm{M}$ is the normalized Maxwellian :

$$
M(k)=C \exp \left(-\frac{\varepsilon(k)}{k_{B} T}\right), \int M(k) d k=1
$$

Here $\varepsilon(k)$ is the energy of carriers, $k_{B}$ is the Boltzmann constant and $T$ the temperature of the crystal. The velocity $v(k)$ depends on the energy band through the relation

$$
v(k)=\frac{1}{\hbar} \nabla_{k} \varepsilon(k) \text {. }
$$

Recent progress in non linear analysis, due mainly to Diperna and Lions [2], allow to obtain a complete existence theory of (1), for boundary value problems [7], [8], as well as for the Cauchy problem [3]. More interesting from a physical point of view is the determination of entropies related to (1). Indeed we have

Proposition 1 Let $\kappa$ be any increasing function, then for every distribution $f$

$$
\int Q(f) \kappa(g) d k \leq 0, \text { with } g=\frac{f}{M(1-f)} .
$$

Define

$$
H(\lambda, k)=\int_{0}^{\lambda} \kappa\left(\frac{s}{M(1-s)}\right) d s
$$

then the functions

$$
S(t, x)=-\int H(f(t, x, k), k) d k
$$

are entropies for (1).

This proposition follows from straightforward algebra using (4), see [3]. One consequence is the determination of equilibrium distributions for $Q$ which are nothing but Fermi-Dirac distributions

$$
F=\frac{1}{1+\exp \left(\frac{\varepsilon(k)-q \mu}{k_{B} T}\right)} .
$$

Concerning the connection between quantum physics and Boltzmann equations, it is well known that it can be provided by tracking wave packets. However, especially for coupling numerical methods, one wish to have a more precise derivation of Boltzmann equations from Bloch equations. With P.A. Markowitch and N.J. Mauser [10], we have introduced a Wigner function approach which is well adapted to describe periodic materials. Namely if $\psi$ is a wave function the related Wigner series is

$$
w(x, k)=\sum_{\mu} e^{i k \mu} \psi(x+\mu / 2) \bar{\psi}(x-\mu / 2)
$$

where $\mu$ belong to the lattice. Then it can be shown that the Wigner function $w$ tends to the distribution function $f$ when the scaled Planck constant and the scaled characteristic length of the lattice vanish. Moreover macroscopic quantities are easily computed from the Wigner function $w$. 


\section{Fluid approximations}

One way to analyse the connection between the Boltzmann equation (1) and fluid models is to use asymptotic analysis. Thus a scaled version of (1) is

$$
\alpha^{2} \partial_{t} f+\alpha\left(v(k) \cdot \nabla_{x} f-E \cdot \nabla_{k} f\right)=Q(f),
$$

where $\alpha$ is a relative value of the mean free path. Then we have to find the limit of the distribution $f$ as $\alpha$ vanishes. In a very general framework, with F. Golse we have proved in [9] that the function $f$ tends to a Fermi-Dirac distribution $F(10)$. The chemical potential $\mu$ solves the drift diffusion equation

$$
\partial_{t} n+\operatorname{divj}=0, n=n(\mu), j=\Pi(\mu) . \nabla_{x}(\mu-V),
$$

where the positive definite matrix $\mu$ is entirely determined by the scattering croos section $s$. The proof relies on entropies estimates based on Proposition 1 and on mean compactness results of [2]. Using parabolic band approximation and relaxation time model, it is possible to compute explicit values of the coefficients in terms of Fermi integrals, [6]. Of course if a non degeneracy assumption is made, we recover the Schockley equation.

Interesting for applications is the analysis of boundary conditions. In first approximation a Dirichlet boundary condition is derived for the concentration. But using such a condition, we neglect a boundary layer. As a concequence non physical discontinuities of the concentration at the boundaries appear in numerical simulations. This boundary layer can be taken into account if the Dirichlet boundary condition $n=n_{0}$ is replaced by a Robin boundary condition

$$
n-\frac{l}{D} j \cdot \nu=n_{0}
$$

where $D$ is a diffusion coefficient, $\nu$ the outward normal and 1 the so-called extrapolation length. This length is also determined by the microscopic scattering mechanisms, see [11].

It seems much more difficult to derive hydrodynamic equations from the Boltzmann equation without $a d-h o c$ assumptions on the distribution function. An attempt is this direction is to introduce an other scaling of the Boltzmann equation which leads to

$$
\alpha\left(\partial_{t} f+v(k) \cdot \nabla_{x} f\right)-E \cdot \nabla_{k} f=Q(f) .
$$

When $\alpha$ tends to 0 , the function $f$ tends to $n F(E, k)$ where $F(E, k)$ is the homogeneous steady state

$$
E . \nabla_{k} F=Q(F), \int F d k=1 .
$$

At a first order of approximation, $\mathrm{n}$ solves the $\mathrm{Ohm}$ law, see [5]. The second order of approximation can only formally be performed. It leads to extended drift-diffuion models [5] or to hydrodynamic systems where the pressure tensor has non isotropic components in the direction of the electric field, [4].

\section{References}

[1] P.A. Markowitch, C.A. Ringhoffer, C. Schmeiser, Semiconductor Equations, Springer, 1990. 
[2] R.J. Diperna, P.L. Lions, Global weak solutions of Vlasov Maxwell systems, Com. Pure Appl. Math. XLII (1989) p 729-757.

[3] F. Poupaud, On a system of non linear Boltzmann equation of semiconductor physics, SIAM J. on Appl. Math. $50 \mathrm{n}^{\circ} 6$ (1990) p 1593-1606.

[4] F. Poupaud, Derivation of hydrodynamic models for semiconductors from the Boltzmann equation, Appl. Math. Lett. 4 n 1 (1991) p 75-79.

[5] F. Poupaud, Runaway phenomena and fluid approximation under high fields in semiconductor kinetic theory, Z. Angew. Math. Mech. 72 (1992) p 359-372.

[6] F. Poupaud, C. Schmeiser, Charge Transport in Semiconductors with Degeneracy Effects, Math. Meth. in Appl. Sci. 14 (1991) p 301-318.

[7] F. Poupaud, Boundary value problems for the stationary Vlasov-Maxwell system, Forum Math. 4 (1992) p 499-527.

[8] A. Nouri, F. Poupaud, Boundary value problems for the stationary VlasovMaxwell-Boltzmann system under Fermi-Dirac statistics, submitted to SIAM J. Math. Anal.

[9] F. Golse, F. Poupaud, Limite fluide des équations de Boltzmann des semiconducteurs pour une statistique de Fermi-Dirac, J. on Asympt. Analysis 6 (1992) p 135-160.

[10] P.A. Markowich, N.J. Mauser, F. Poupaud, A Wigner Function Approach to (Semi)classical Limits : Electrons in a Periodic Potential, submitted to J. of Math. Phys.

[11] F. Poupaud, A. Yamnahakki, Conditions limites du second ordre pour l'équation de dérive diffusion des semiconducteurs, Internal Report 330 (1993), Lab. J.A. Dieudonné, Univ. de Nice. 